\title{
Electron Holographic Observation of a Micro-Magnetic Field Current Generated from a Helical Carbon Coil
}

\author{
K. Yamamoto, ${ }^{*}$ T. Hirayama,** M. Kusunoki,** S. Yang***, S. Motojima*** \\ * Japan Society for the Promotion of Science, c/o Japan Fine Ceramics Center. \\ ** Japan Fine Ceramics Center, 2-4-1, Mutsuno, Atsuta-ku, Nagoya, 456-8587 Japan. \\ *** Department of Applied Chemistry, Gifu University, Gifu 501-1193 Japan.
}

In 1990, Motojima et al. [1] succeeded in producing regularly helical carbon coils using a chemical vapor deposition (CVD) method. Applications of such coils have been postulated for various fields like micro-mechanics and electromagnetic sensors, and more recently for medical science. Some properties of these carbon coils have been examined for application, such as the mechanical [2], electrical [3], chemical [4], and electromagnetic wave absorption [5] properties. In this report we describe our investigation of whether these coils are suitable for use as micro-solenoids in magnetic devices. When a direct current is supplied to the coil, it generates a micro-magnetic field. To utilize the micro-magnetic field in magnetic devices, the magnetic field must be observed directly and quantitatively. We were recently able to observe it using a focused ion beam (FIB) system and electron holography. We report the details of the specimen preparation and the magnetic field observation.

To supply direct current to the carbon coil in a TEM, we connected the coil to two electrodes fixed in a TEM specimen holder. We used an FIB system with a fine tungsten probe (Hitachi FB-2100 micro-sampling system) for manipulating and connecting the coil. Figure 1 shows a scanning ion microscopy image of a carbon coil connected to the electrodes (gold wire). The electrodes and the coil were connected by a tungsten deposition in the FIB system. The coil was coated with gold so that its electric resistance was decreased to generate a magnetic field strong enough to be detected.

A hologram was taken of the area indicated by the dashed lines in Fig. 1, after a 14.0-mA direct current was supplied to the coil. Figure 2 (a) shows the phase image reconstructed from the hologram. The micro-magnetic field generated by the coil was observed directly. The arrows indicate the direction of the magnetic flux. To analyze the magnetic field quantitatively, we assumed a cylindrical magnet model instead of the current-supplied coil, and calculated the two-dimensional magnetic field around it. Figure 2 (b) shows an image of the calculated field. This calculation takes into account the reference wave distortion caused by the magnetic field from the coil. The 
calculated image agrees well with the experimental image.

In summary, a helical carbon coil was connected to small electrodes using the FIB micro-sampling technique. The micro-magnetic field current generated from the coil was then quantitatively observed by electron holography. Computer simulation of electron holography was also performed to analyze the field, and the resulting image agreed well with the experimental image. Therefore, the helical carbon coils are good candidates for use as micro-solenoids, and FIB and electron holography are useful techniques for examining such micro-magnetic devices.

\section{References}

[1] S. Motojima, M. Kawaguchi, K. Nozaki, H. Iwanaga, Appl. Phys. Lett. 56 (1990) 321.

[2] S. Motojima, S. Ueno, T. Hattori, H. Iwanaga, J. Cryst. Growth, 96 (1989) 383.

[3] K. Kaneto, M. Tsuruta, S. Motojima, Synthetic Metals, 103 (1999) 2578.

[4] S. Motojima, W. I. Hwang, T. Kuzuya, H. Iwanaga, J. Mater. Sci., 36 (2001) 71.

[5] S. Motojima, T. Hamamoto, N. Ueshima, Y. Kojima, H. Iwanaga, Electrochem. Soc. Proc., 25 (1997) 433.

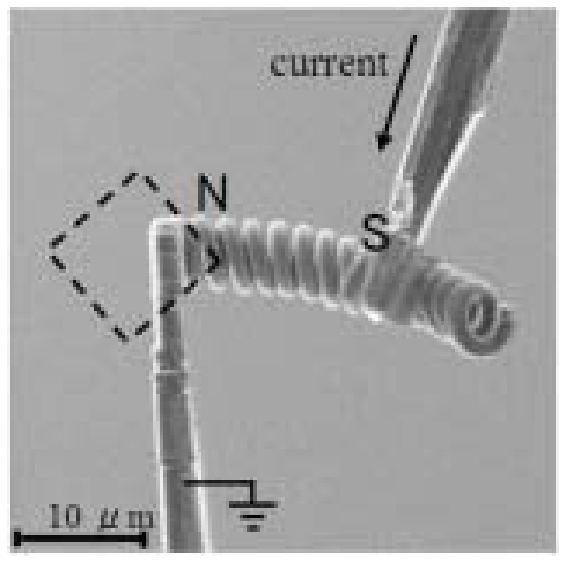

Fig. 1 Scanning ion microscopy image of helical carbon coil connected to two gold electrodes.
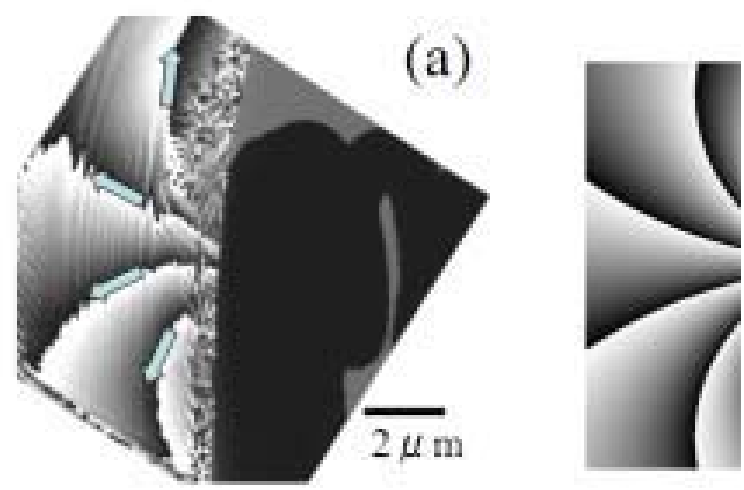

(b)

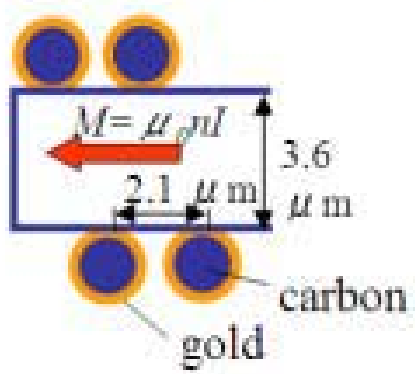

Fig.2 Phase images around helical carbon coil: (a) experimental image with 14.0-mA current, (b) simulated image. 